\title{
Electronic circuit Emuling a first-order time-delay differential equation
}

\author{
C. Jiménez-Calvillo ${ }^{a, *}$, I. Campos-Cantón ${ }^{b}$ and L. J. Ontañón-García ${ }^{c}$ \\ ${ }^{a}$ Instituto de Investigación en Comunicación Óptica, Universidad Autónoma de San Luis Potosí. \\ Alvaro Obregón 64, 78000 San Luis Potosí, SLP, México. \\ *e-mail: cesar.jimenez@uaslp.mx \\ ${ }^{b}$ Facultad de Ciencias, Universidad Autónoma de San Luis Potosí. \\ Alvaro Obregón 64, 78000 San Luis Potosí, SLP, México. \\ ${ }^{c}$ Coordinación Académica Región Altiplano Oeste, Universidad Autónoma de San Luis Potosí. \\ Carretera Salinas-Santo Domingo 78600 ,Salinas de Hidalgo, SLP, México.
}

Received 19 January 2021; accepted 7 July 2021

\begin{abstract}
This article provides undergraduates a useful tool for a better understanding of the time delay effect on an electronic circuit. The time delay effect is analyzed in this paper a first-order differential equation. This linear time delay is associated with the amplitude of a firstorder differential equation and is responsible for three responses: one of the responses is a differential equation type in first-order without delay, another one of the responses is a differential equation type in second-order and finally, we have the response of a harmonic oscillator. The proposed circuit is an emulator that develops the three different responses mentioned above. Simulink-Matlab software was used to implement the time delay and simulate the differential equation. These simulation results coincide with the theoretical results. In the same manner, the experimental results match those of the theory. The electronic circuits suggested consist of three blocks: an integrator block, a phase shift block, and a gain block. The electrical circuit is composed of resistors, capacitors, and operational amplifiers.
\end{abstract}

Keywords: Time delay; phase shift; analog circuits; differential equation; circuit theory.

DOI: https://doi.org/10.31349/RevMexFisE.19.010202

\section{Introduction}

The model that replicates the best behavior must take the last system state into account. That is to say, the use of the timedelay differential equation is a good prospect for this purpose. For instance, population dynamics are supposed to have responsive interaction between two different organisms, hence the immediate response due to the presence of aggression. However, we have to consider the time it takes to get this response; for instance, plants that are attacked by herbivores need time to recover their foliage, and the predator needs time to consume its prey as well, etc. In addition, populations generally do not asymptotically reach their equilibrium point but rather oscillate around it. This behavior can be modeled by adding time-delays in the reaction time. The time-delays differential equation is suitable for modeling these cases; in general, they can be very complicated, although in certain situations, it is possible to perform fairly complete qualitative or geometrical research.

The study of time-delay systems has been of great interest in the science field. The industrial and investigation systems usually need to include delay effects in their processes to acquire excellent performance. To model Thermal effects, we must always consider the effects provided by time-delay for a better approximation. In electronics, a time-delay is considered in design and fabrication systems [1]. Other examples are feedback control [1], linear system stabilization [2], signal research, noise modeling effects in radio frequency (RF), and all-pass filters [3-5]. So far, most of the existing results reported on time-delay are theoretical [6], and normally no circuit is built.

On this task, and to teach students of science and engineering, we developed experiments for time-delay processes and implemented an emulator electronic circuit. for a timedelay device. We created a solution for each time interval in which the behavior changed, hence obtaining the conditions for stability. The acquired results are numerically shown on the Matlab software and experimentally implemented in the emulator circuit.

This article is addressed as follows: in Sec. 2, a solution for time-delay first-order differential equation is given. In Sec. 3, the suggested emulator circuit is developed through Kirchhoff's laws. In Sec. 4, the numerical solutions tested in MatLab are shown as well as experimental results of the circuit. In the $\mathrm{AC}$ and $\mathrm{DC}$ section, the $\mathrm{AC}, \mathrm{DC}$, and transient analysis for each stage of the circuit is developed, and discussion and conclusions are presented in Sec. 5.

\section{Firts order differential equation with time delay}

Based on the first-order differential equation with time-delay [7], we infer possible solutions with a time-delay $(\tau)$. Technically, if we give $\tau$ a value, we can have different solutions: solution in a first-order system without time-delay, the damped solution from a second-order without time-delay, and an oscillatory response. 
The time-delay differential equation theory is concerned with the variation of the state of $x$ which depends on time instant $t$, and the previous moment $\tau$.

$$
\dot{x}(t)=f\left(t, x(t), x\left(t-\tau_{1}\right), \cdots, x\left(t-\tau_{n}\right)\right),
$$

where $f$ is a given function, and time delay $\tau_{j}$ satisfies $\tau_{j}>0$.

A particular case of a time-delay first-order differential equations with constant coefficients, is when only one timedelay exists:

$$
\dot{x}(t)=f(t, x(t), x(t-\tau)),
$$

On this task, we researched the next differential equation, with amplitude $a$, time-delay $\tau$, and a $g(t)$ prefunction.

$$
\left.\begin{array}{l}
\dot{x}(t)=a x(t-\tau), \text { in }[0, b], b>0 \\
x(t)=g(t) \text { in }[-\tau, 0]
\end{array}\right\}
$$

where $\{a, b, \tau\} \in \mathbb{R}$.

Equation (3) is the simplest concept to teach a student in the science field.

\subsection{Characteristic equation}

Assume an exponential solution in this way

$$
x(t)=c e^{z t},
$$

where $z \in \mathbb{Z}$.

Differentiating (4) concerning time,

$$
\dot{x}=c z e^{z t},
$$

and substituting (4) and (5) in (3)

$$
z=a e^{-z \tau},
$$

the characteristic equation is

$$
z e^{z \tau}-a=0 .
$$

\subsection{Solution}

Therefore, the objective is to find the roots of (7); or its equivalent equation

$$
(p+i q) e^{(p+i q) \tau}-a=0
$$

from where we get the real and imaginary part

$$
\begin{gathered}
\text { Real } \rightarrow e^{p \tau}(p \cos q \tau-q \sin q \tau)-a=0, \\
\text { Imaginary } \rightarrow e^{p \tau}(p \sin q \tau+q \cos q \tau)=0,
\end{gathered}
$$

from (10) we get $p$

$$
p=-q \cot q \tau,
$$

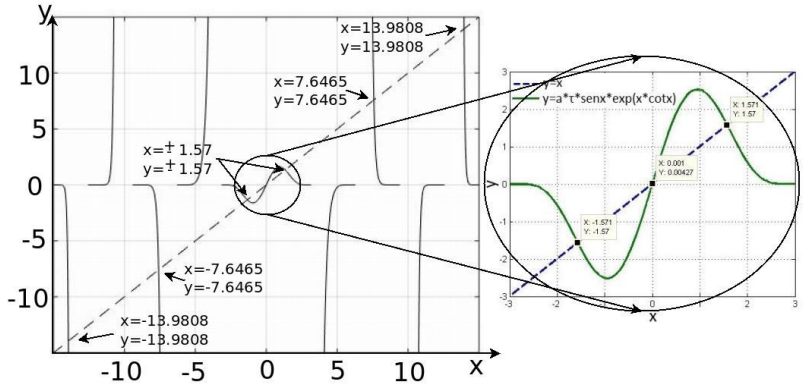

FIGURE 1. The solution is satisfied in the cross point of two curves, in this case at interval -15 to 15 .

substituting (11) in (9)

$$
q=-a \sin q \tau e^{q \tau \cot q \tau},
$$

multiplying (12) by $\tau$ and changing the variable in $x=q \tau$,

$$
x=-a \tau \sin x e^{x \cot x},
$$

to resolve (13), we find intersection of $y=x$ and $y=$ $-a \tau \sin x e^{x \cot x}$; therefore, when the values on the left side of the equation coincide with those on the right side in (13), the solution has been found. Figure 1 it shows this situation.

Figure 1 illustrates an infinity amount of solutions, those solutions are found in each intersection; for instance, in $x= \pm \pi / 2$. The solutions are numerically found.

Now, $x_{k}$ is one root in Eq. (13), as $q=x / \tau$, be $q_{k}=$ $x_{k} / \tau$. The $p_{k}$ values are obtained using the $q_{k}$ values in Eq. (11). Then, the roots of (7) are $p_{k}+i q_{k}$, and the characteristic solutions are $e^{p_{k}} \cos \left(q_{k} t\right)$ and $e^{p_{k}} \sin \left(q_{k} t\right)$. As in Eq. (3) is linear, the formal solution is

$$
x(t)=\sum_{k=0}^{\infty} e^{p_{k}}\left[c_{1 k} \cos \left(q_{k} t\right)+c_{2 k} \sin \left(q_{k} t\right)\right] .
$$

where $c_{1 k}$ and $c_{2 k}$ are constants.

\subsubsection{Looking for a solution}

From Fig. 1, if $x=0$ and $q \neq 0$ then $\tau=0$, that corresponds to a system without time-delay, on the other hand, if $x=\pi / 2$ and using (11) and (12)

$$
\begin{aligned}
& p=-q \cot \pi / 2=0 \\
& q=-a \sin \pi / 2 e^{\pi / 2 \cot \pi / 2}=-a
\end{aligned}
$$

thus $z=-i a$ and $x=c e^{-i a t}$, concerning an oscillatory system, then, your solution is given by (14). The relationship between $a$ and $\tau$ is obtained from (13),

$$
a \tau=-\pi / 2 .
$$

Now, if $x$ is small, we approximate $\sin x$ to $x, \cos x$ to 1 , and $\cot x$ to $1 / x$. Under these assumptions, from (11) and (12) $p=-1 / \tau, q=0$, because if we take the limit in Eq. (11)

$$
\lim _{q \rightarrow 0}(-q \cot q \tau)=\lim _{q \rightarrow 0} \frac{-q \tau \cot q \tau}{\tau \sin q \tau}=-\frac{1}{\tau},
$$


and applying it to (13)

$$
a \tau=-\frac{1}{e} .
$$

Thus $z=-1 / \tau$ and $x=c e^{-\frac{1}{\tau} t}$, concerning to a first order system without delay if $-(1 / e)<a \tau<0$.

Then, in this case, the general solution is given by

$$
x(t)=c_{0} e^{-\frac{t}{\tau}}+\sum_{k=1}^{\infty} e^{p_{k}}\left[c_{1 k} \cos \left(q_{k} t\right)+c_{2 k} \sin \left(q_{k} t\right)\right] .
$$

If we take values in $-(\pi / 2)<a \tau<-(1 / e)$, then $p \neq 0$ and $q \neq 0$. Thus $z=-p+i q$ and $x=c e^{-(p+i q) t}$. Getting the answer for a second-order underdamped system. Then, the solution is given by (14). For example, if $x=\pi / 4$ and using the change in a variable in (11), we then have these values for $p$ and $q$

$$
\begin{aligned}
& p=-\frac{\pi}{4 \tau} \\
& q=\frac{\pi}{4 \tau}
\end{aligned}
$$

thus

$$
z=-\frac{\pi}{4 \tau}(1-i)
$$

and

applying (13)

$$
x=c e^{-\frac{\pi}{4 \tau}(1-i) t},
$$

$$
a \tau=-\frac{\pi \sqrt{2}}{4 e^{\frac{\pi}{4}}} .
$$

\subsection{Approximate solution}

We can give an approximate solution for (17), defining the function $x_{n}(t)$ in the following manner:

$$
\begin{gathered}
\text { If }-(1 / e)<a \tau<0 \\
x_{n}(t)=c_{0} e^{-\frac{t}{\tau}}+\sum_{k=1}^{n} e^{p_{k}}\left[c_{1 k} \cos \left(q_{k} t\right)+c_{2 k} \sin \left(q_{k} t\right)\right] . \\
\text { If } a \tau<-\frac{1}{e} \\
x_{n}(t)=\sum_{k=1}^{n} e^{p_{k}}\left[c_{1 k} \cos \left(q_{k} t\right)+c_{2 k} \sin \left(q_{k} t\right)\right] .
\end{gathered}
$$

to guarantee the continuity of the solution in $t=0$ is required that $x_{n}(0)=g(0)$ to be able to calculate its coefficients $c_{i}$, the interval $[-\tau, 0]$ is split into subintervals in which the function of $x_{n}(t)$ and the pre-function of $g(t)$ are evaluated as $x_{n}\left(t_{i}\right)=g\left(t_{i}\right)$.

\subsubsection{Illustration as First Order type}

Take the following values: $a=1, \tau=1 / 2 e$ and $g(t)=e^{-t^{2}}$, we find intersection of $y=x$ and $y=-a \tau \sin x e^{x \cot x}$ those correspond to $x= \pm 7.3845, \pm 13.8481$, for instance, using the negative values of $q=x / \tau=-40.146,-75.286$ and $p=-q \cot q \tau=-20.367,-22.390$. We build an approximate solution for $n=2$, and (19) is expressed as

$$
x_{2}(t)=c_{0} e^{-\frac{t}{\tau}}+\sum_{k=1}^{2} e^{p_{k}}\left[c_{1 k} \cos \left(q_{k} t\right)+c_{2 k} \sin \left(q_{k} t\right)\right]
$$

subdividing the interval $[-\tau, 0]=[-(1 / 2 e), 0]$ in four parts, to calculate $t_{i}$ instants necessary to solve $c_{i}$ constants. These are $t=0,-0.0459,-0.0919,-0.1379,-0.1839$, and the corresponding $c_{i}$ are: $c_{0}=0.7740, c_{11}=0.0230, c_{21}=$ $0.1598, c_{12}=0.2029$ and $c_{22}=-0.1896$. Then $x_{2}(t)$ is:

$$
\begin{aligned}
x_{2}(t) & =0.7740 e^{-2 e t}+e^{-20.367 t}(0.0230 \cos [-40.146 t] \\
& +0.1598 \sin [-40.146 t]) \\
& +e^{-22.390 t}(0.2029 \cos [-75.286 t] \\
& -0.1896 \sin [-75.286 t]) .
\end{aligned}
$$

The results are shown in Fig. 2a.

\subsubsection{Illustrating a Second Order type}

Taking the next values: $a=1, \tau=1$ and $g(t)=e^{-t^{2}}$, we found that $x= \pm 1.3361, \pm 7.5921$, using negative values $q=-1.3361,-7.5921$ and $p=-0.3194,-2.0350$. For $n=2,(20)$ is expressed as
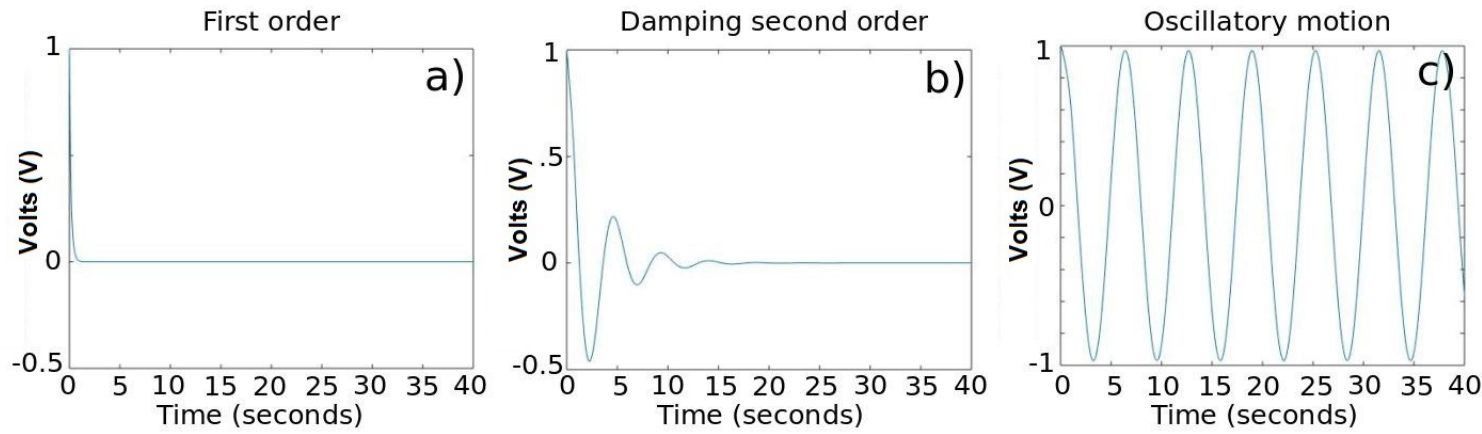

FIGURE 2. Approximate solution: a) first-order, b) second-order, c) oscillation motion. 


$$
x_{2}(t)=\sum_{k=1}^{2} e^{p_{k}}\left[c_{1 k} \cos \left(q_{k} t\right)+c_{2 k} \sin \left(q_{k} t\right)\right]
$$

subdividing the interval $[-\tau, 0]=[-1,0]$ into three parts, to calculate $t_{i}$ instants: $t=0,-0.3333,-0.6667,-1$, and the corresponding $c_{i}$ are: $c_{11}=0.9686, c_{21}=-0.0962$, $c_{12}=0.0313$ and $c_{22}=0.0168$. Then $x_{2}(t)$ is:

$$
\begin{aligned}
x_{2}(t) & =e^{-0.3194 t}(0.9686 \cos [-1.3361 t] \\
& -0.0962 \sin [-1.3361 t]) \\
& +e^{-2.0350 t}(0.0313 \cos [-7.5921 t] \\
& +0.0168 \sin [-7.5921 t]) .
\end{aligned}
$$

The results are shown on Fig. $2 b$.

\subsubsection{Illustration as Oscillatory type}

Taking the next values: $a=1, \tau=\pi / 2$ and $g(t)=e^{-t^{2}}$, we found that $x= \pm 1.5708, \pm 7.6465$, using negative values $q=-1,-4.8679$ and $p=0,-1.0247$. For $n=2$, (20) is expressed as

$$
x_{2}(t)=\sum_{k=1}^{2} e^{p_{k}}\left[c_{1 k} \cos \left(q_{k} t\right)+c_{2 k} \sin \left(q_{k} t\right)\right],
$$

subdividing the interval $[-\tau, 0]=[-\pi / 2,0]$ into three parts, to calculate $t_{i}$ instants: $t=0,-0.5236,-1.0472,-1.5708$, and the corresponding $c_{i}$ are: $c_{11}=0.9646, c_{21}=-0.1130$, $c_{12}=0.0354$ and $c_{22}=0.0330$. Then $x_{2}(t)$ is:

$$
\begin{aligned}
x_{2}(t) & =0.9646 \cos (-1 t) \\
& -0.1130 \sin (-1 t)+ \\
& e^{-1.0247 t}(0.0354 \cos [-4.8679 t] \\
& +0.0330 \sin [-4.8679 t]) .
\end{aligned}
$$

The results are shown on Fig. 2c.

With these results, an electronic circuit is designed. With the electronic circuit designed, students can connect the abstract concept with a real system and adjust the time delay in the suggested circuit.

\section{Time-delay emulator circuit}

The objective is to design a time-delay emulator electronic circuit based on (3); for this, we are considering two parts in this equation: left side and right side of the equation. The left side has been taken as a first-order differential equation without delay (see Fig. 3a), and the right side corresponds to the delayed signal (Fig. 3b). Since the left and right-hand sides are equal, this creates a closed-loop, as is shown in Fig. 3c.
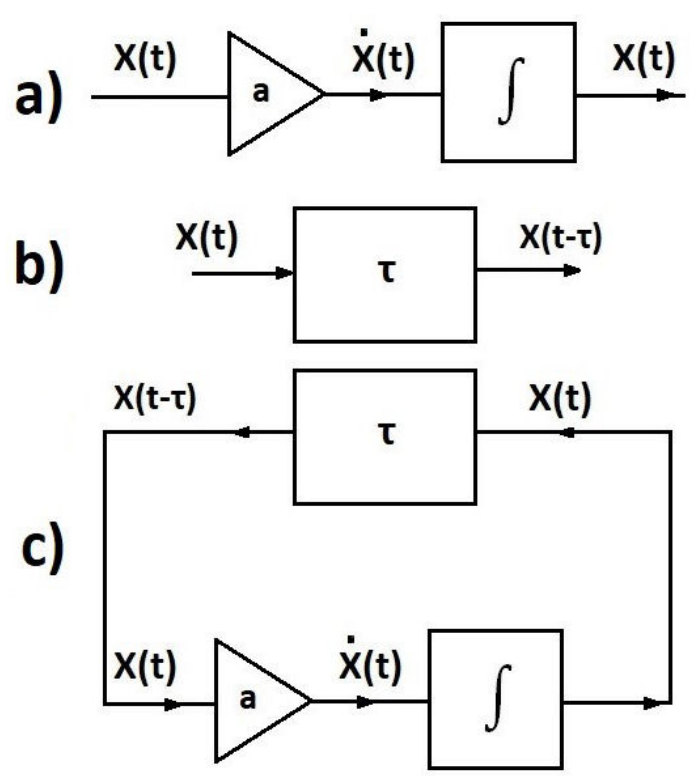

FIGURE 3. Time-delay block diagram: a) first-order differential equation without delay $(\dot{x}(t)=a x(t))$, b) delayed signal $(x(t-\tau)), \mathrm{c})$ close loop.

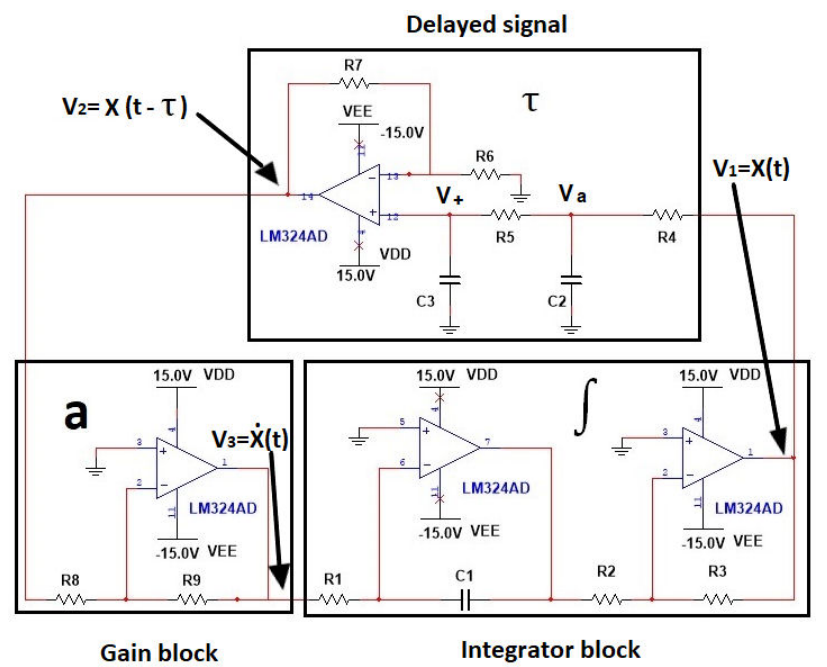

FIGURE 4. The proposal electronic circuit is comprised of: gain circuit " $a$ ", integrated circuit " $\int$ " and time-delay circuit " $\tau$ ".

Considering the block diagram in Fig. 3, the next electronic circuit is suggested in Fig. 4 . Where the voltages $v_{1}$, $v_{2}$, and $v_{3}$ take the role as the variables $x(t), x(t-\tau)$, and $\dot{x}(t)=a x(t-\tau)$, respectively. The time delay in electronics circuits is mapped as phase-angle shift $(\tau=\theta)$.

Using Kirchhoff laws in the electronic circuit

$$
\begin{aligned}
& v_{1}=\frac{R_{3}}{R_{1} R_{2} C_{1}} \int v_{3} d t, \\
& v_{3}=-\frac{R_{9}}{R_{8}} v_{2},
\end{aligned}
$$


and $v_{2}$ as impedance function, considering $R=R_{4}=R_{5}$, $C=C_{2}=C_{3}, Z=(1 / i \omega C)$,

$$
v_{2}=\left(\frac{R_{7}}{R_{6}}+1\right) \frac{Z^{2}}{R^{2}+3 R Z+Z^{2}} v_{1},
$$

and expressed as frequency

$$
v_{2}=\left(\frac{R_{7}}{R_{6}}+1\right) \frac{\left(1-R^{2} C^{2} \omega^{2}\right)+i 3 R \omega}{\left(1-R^{2} C^{2} \omega^{2}\right)^{2}+9 R^{2} C^{2} \omega^{2}} v_{1} .
$$

from where the magnitude ratio and phase-angle shift

$$
\begin{aligned}
\left|v_{2} / v_{1}\right| & =\frac{\frac{R_{7}}{R_{6}}+1}{\left(1-R^{2} C^{2} \omega^{2}\right)^{2}+9 R^{2} C^{2} \omega^{2}} \\
& \times \sqrt{R^{4} C^{4} \omega^{4}+7 R^{2} C^{2} \omega^{2}+1} \\
\theta & =\arctan \frac{3 R C \omega}{1-R^{2} C^{2} \omega^{2}} .
\end{aligned}
$$

The circuit is easy to implement by students since this only consists of operational amplifiers, resistors, and capacitors.

\section{Numeric and Experimental Results}

First, we carried out a Matlab-Simulink simulation of timedelayed block diagram Fig. 3, using the parameters in Table I. The result is shown in Fig. 5.

The oscillatory results, Fig. 5c), have a frequency of 1 Hz. Using this frequency and the parameters of Table I $(a, \tau)$, we calculated the resistor and capacitance values on the suggested electronic circuit.

\section{Gain a}

As $v_{3}=a x(t-\tau)$ and $v_{2}=x(t-\tau)$, we see from (22) that $a=-\left(R_{9} / R_{8}\right)$, if we take $R_{8}=R_{9}=10 \mathrm{~K} \Omega$, then $a=-1$.

\section{Block Integrator}

It proceeds as follows: to calculate $R_{1}, R_{2}, R_{3}$, and $C_{1}$. Let $v_{3}=\cos \omega t$, and using (21),

$$
v_{1}=\frac{R_{3} \sin \omega t}{R_{1} R_{2} C_{1} \omega}
$$

from which we adjust $\left(R_{3} / R_{1} R_{2} C_{1} \omega\right)$ to acquire a unity gain. Like $f=1 \mathrm{~Hz}$, and if we take $R_{2}=R_{3}=10 \mathrm{~K} \Omega$ and $C_{1}=1 \mu \mathrm{F}$, we get $R_{1}=1 \mathrm{M} \Omega$.

\section{TABLE I. Parameters.}

\begin{tabular}{ccc}
\hline $\mathrm{V}$ (Volts) & $\tau(\mathrm{sec})$. & response \\
\hline-1 & $\frac{1}{2 e}$ & first order \\
-1 & 1 & damping second order \\
-1 & $\frac{\pi}{2}$ & oscillatory motion \\
\hline
\end{tabular}
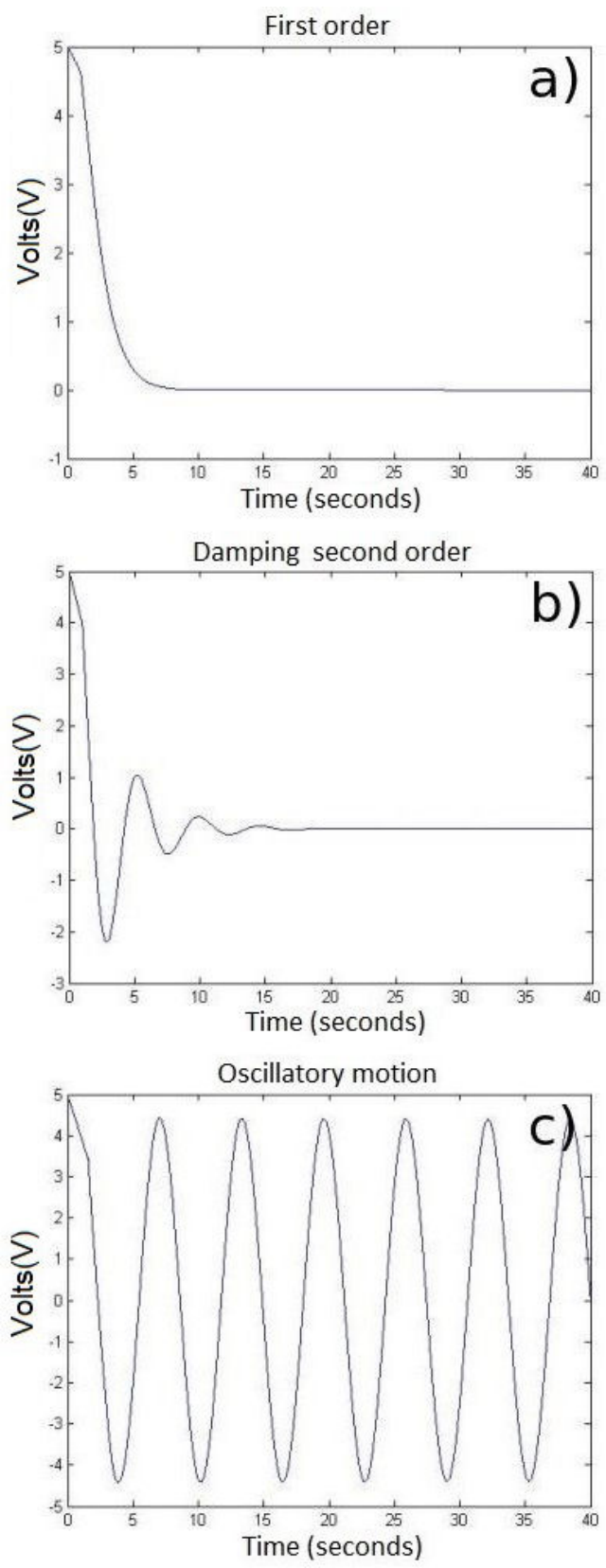

FIGURE 5. Response: a) first-order, b) damping second-order, c) oscillatory motion.

\section{Time-delay}

Now, to calculate $R=R_{4}=R_{5}$, we need propose $C=C_{2}=C_{3}$. From (26),

$$
R=\frac{-3 \pm \sqrt{9+4 \tan ^{2} \theta}}{2 C \omega \tan \theta},
$$

and using $C=1 \mu \mathrm{F}$, and the fact that time-delay is directly proportional to the phase $(\tau=\theta$ ), we get $R$ values to adjust $\theta$; these are shown in Table IIcolumn two.

\section{Magnitude}

Finally, to calculate $R_{7}$, we propose $R_{6}$. The magnitude of (25) must be adjusted to unity; for this purpose, we get $R_{7}$ of this equation, 


\begin{tabular}{ccc}
\hline \hline \multicolumn{2}{l}{ TABLE II. Magnitude and phase. } & \\
\hline$\theta$ & $R$ & $R_{7}$ \\
\hline$\frac{1}{2 e}(\mathrm{rad})$ & $59.12 \mathrm{~K} \Omega$ & $121.65 \Omega$ \\
$1(\mathrm{rad})$ & $425.25 \mathrm{~K} \Omega$ & $5.16 \mathrm{~K} \Omega$ \\
$\frac{\pi}{2}(\mathrm{rad})$ & $1 \mathrm{M} \Omega$ & $20 \mathrm{~K} \Omega$ \\
\hline
\end{tabular}

$$
R_{7}=\left(\frac{\left(1-R^{2} C^{2} \omega^{2}\right)^{2}+R^{2} C^{2} \omega^{2}}{\sqrt{R^{4} C^{4} \omega^{4}+7 R^{2} C^{2} \omega^{2}+1}}-1\right) R_{6},
$$

and using $R_{6}=10 \mathrm{~K} \Omega, C=1 \mu \mathrm{F}$, and $R$ given in Table II column two, we get $R_{7}$ values as are shown in Table II column three.

With these calculated values, the experiment is performed, and the results are shown in Fig. 6.

\section{AC and DC block analysis}

$\mathrm{AC}, \mathrm{DC}$, and noise response analysis are important in the design process, as one can see the properties of electronic circuits manufactured. For this reason, we carried out this study in this subsection. Based on the circuit shown in Fig. 4 and Table III, its analysis by blocks is carried out.

\subsection{AC analysis}

\section{Integrator block}

Using (21) its transfer function is

$$
G_{\text {int }}=\frac{R_{3}}{R_{1} R_{2} C_{1} S}
$$

and taking the values of resistors and capacitor given above, its magnitude drops $20 \mathrm{~dB}$ per decade, its phase remains constant at -90 degrees, and its cutoff frequency is given at 0.22 $\mathrm{Hz}$

\section{Gain block}

Using (22) its transfer function is

$$
G_{\text {gain }}=-\frac{R_{9}}{R_{8}}
$$

and taking the values of resistors given above, its magnitude has unity gain per decade, and its phase remains constant at 180 degrees.

\section{Delayed block}

Using (23) its transfer function is

$$
G_{\text {delay }}=\left(\frac{R_{7}}{R_{6}}+1\right) \frac{1}{R^{2} C^{2} S+3 R C S+1},
$$

and taking the values of resistors and capacitors given above, its Bode plot is shown in Fig. 7. Where: for Fig. 7 its magnitude drops $-40 \mathrm{~dB}$ per decade, its phase go to -180 degrees, and its cutoff frequency is given at $1.11 \mathrm{~Hz}$ for $R=59.12 \mathrm{k} \Omega$ and $R_{7}=121.65 \Omega$, for Fig. 8 its magnitude drops $-40 \mathrm{~dB}$

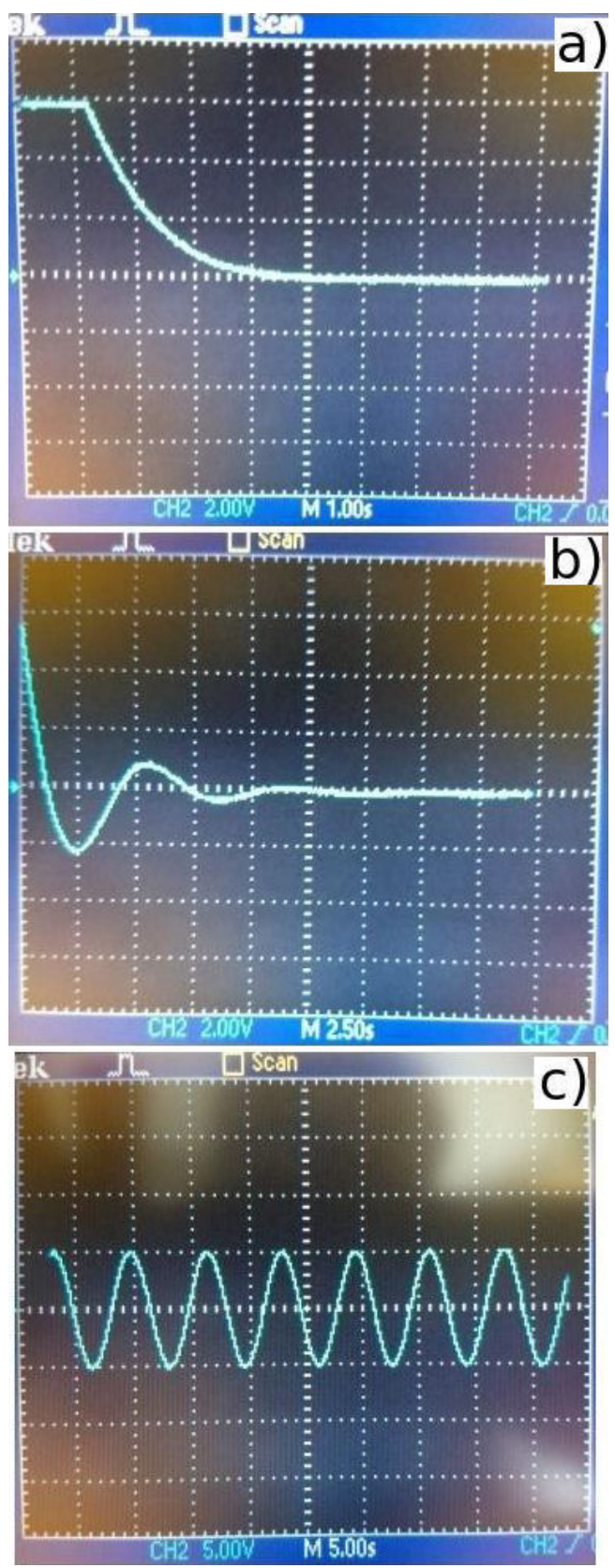

FIGURE 6. Response: a) first-order, b) damping second-order, c) oscillatory motion.

per decade, its phase go to -180 degrees, and the cutoff frequency is given at $0.286 \mathrm{~Hz}$ for $R=425.25 \Omega$ and $R_{7}=5.16 \mathrm{~K} \Omega$, and finally for Fig. 9 its magnitude drops $-40 \mathrm{~dB}$ per decade, its phase go to -180 degrees, and its cutoff frequency is given at $0.214 \mathrm{~Hz}$ for $R=1 \mathrm{M} \Omega$ and $R_{7}=20 \mathrm{~K} \Omega$.

\subsection{DC analysis}

For the DC analysis, we use the step signal as input to observe the transient and steady-state response at each block. 


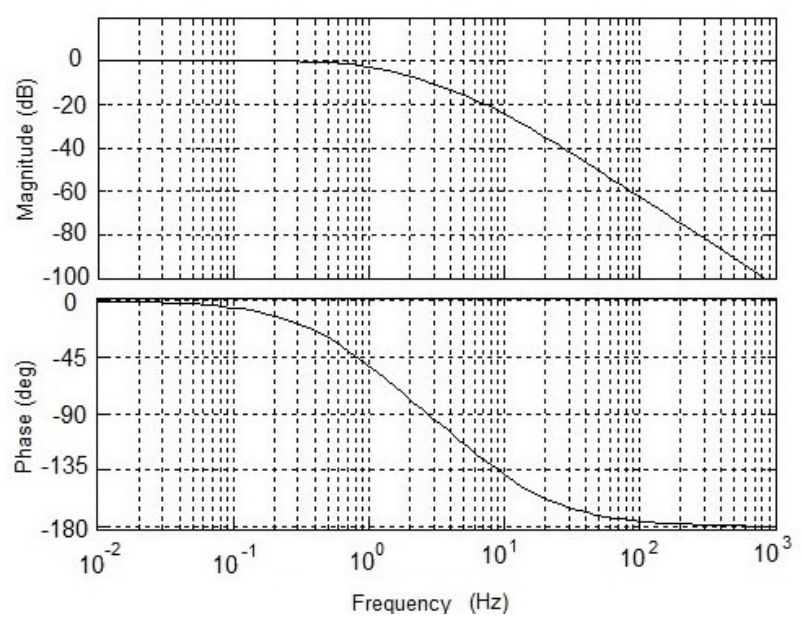

FIGURE 7. Frequency analysis: a) For values of $R=59.12 \mathrm{~K} \Omega$ and $R_{7}=121.65 \Omega$.

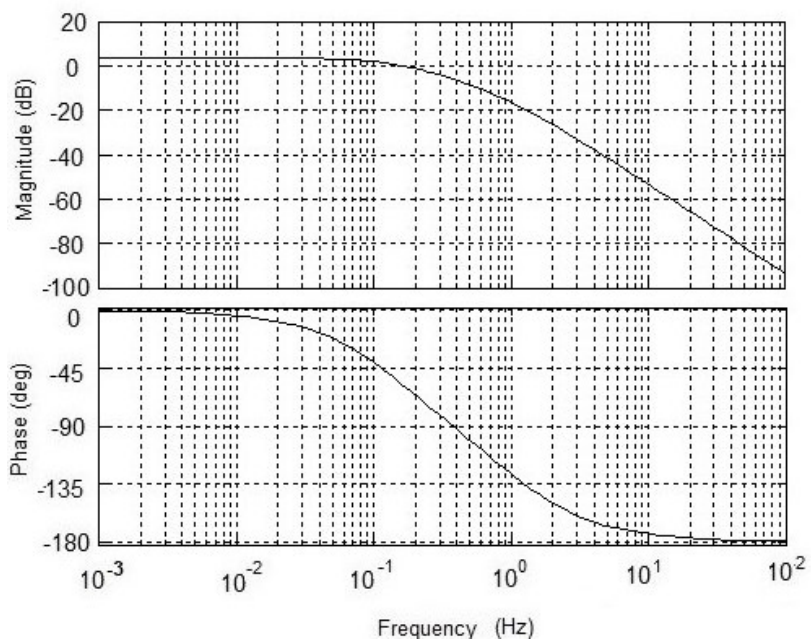

FIGURE 8. Frequency analysis: b) For values of $R=425.25 \mathrm{~K} \Omega$ and $R_{7}=5.16 \mathrm{~K} \Omega$.

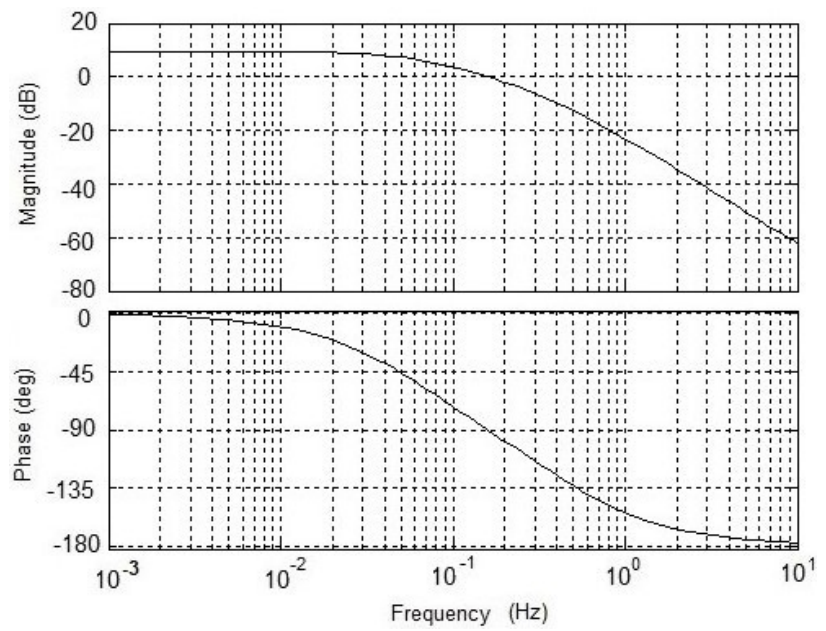

FIGURE 9. Frequency analysis: c) For values of $R=1 \mathrm{M} \Omega$ and $R_{7}=20 \mathrm{~K} \Omega$.

\section{Integrator block}

For the integrator block, we use the transfer function (27) and taking values of resistors given above, the step response corresponds to a straight line with slope 1 , as expected for the integration function.

\section{Gain block}

For the gain block, we use the transfer function (28) and taking values of resistors given above, we obtain a gain of $a=-1$.

\section{Delayed block}

For delayed block we use the transfer function (29) and taking values of resistors and capacitor given above, its step response is shown in Fig. 10. Where: the curve a) corresponds to a critically damped response for $R=59.12 \Omega$ and $R_{7}=121.65 \Omega$; for the curve b) corresponds to an overdamped response for $R=425.25 \Omega$ and $R_{7}=5.16 \mathrm{~K} \Omega$, and in the same case for the curve, c) we have an overdamped response for $R=425.25 \Omega$ and $R_{7}=5.16 \mathrm{~K} \Omega$.

The theoretical solution is found by calculating the transient and steady-state response. For this, we make use of the voltage on capacitors $\mathrm{C} 2$ and $\mathrm{C} 3$ and finally add the gain $\left(R_{7} / R_{6}+1\right)$, where the following equations are found.

Steady state response:

$$
V_{C 2}=V_{C 3}=V_{1} .
$$

Transient response: The equation in $C_{2}$ and $C_{3}$ are

$$
\begin{aligned}
\frac{d V_{C 2}}{d t} & =-\frac{1}{C_{2} R_{4} R_{5}} V_{C 2}+\frac{V_{1}}{R_{4}}+\frac{V_{C 3}}{R_{5}}, \\
\frac{d V_{C 3}}{d t} & =-\frac{V_{C 3}}{R_{5} C_{3}}+\frac{V_{C 2}}{R_{5} C_{3}} .
\end{aligned}
$$

Solving for the total response for $R_{4}=R_{5}=59.12 \mathrm{~K} \Omega$, $R_{7}=121.65 \Omega$ and $C_{2}=C_{3}=1 \mu \mathrm{F}$. We obtain:

$$
V_{C 3}=-e^{-33.83 t}-e^{-16.91 t}+e^{-33.83 t}-e^{-16.91 t}+1,
$$

and for $R_{4}=R_{5}=425.25 \mathrm{~K} \Omega, R_{7}=5.16 \mathrm{~K} \Omega$ and $C_{2}=C_{3}=1 \mu \mathrm{F}$.

$$
V_{C 3}=-e^{-4.703 t}-e^{-2.352 t}+e^{-4.703 t}-e^{-2.352 t}+1 \text {, }
$$

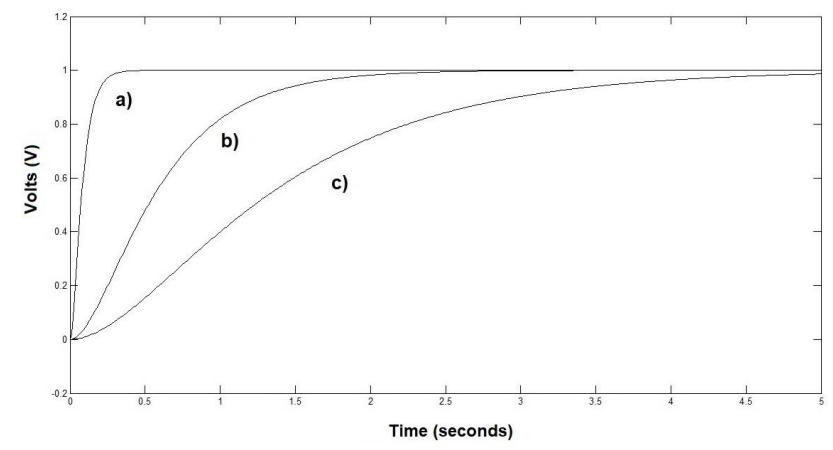

FIgURE 10. Step response for: a) $R=59.12 \Omega$ and $R_{7}=121.65 \Omega$, b) $R=425.25 \Omega$ and $R_{7}=5.16 \mathrm{~K} \Omega$, c) $R=425.25 \Omega$ and $R_{7}=5.16 \mathrm{~K} \Omega$. 
TABLE III. SNR, THD and SNAD values for different noise levels.

\begin{tabular}{cccc}
\hline$s($ Vrms $)$ & SNR $(\mathrm{dB})$ & $T H D(\mathrm{~dB})$ & $\mathrm{SNAD}(\mathrm{dB})$ \\
\hline 0.007 & 36.98 & -6.02 & 6.01 \\
0.070 & 16.98 & -6.02 & 5.68 \\
0.353 & 3.01 & -6.02 & 1.24 \\
\hline
\end{tabular}

finally for $R_{4}=R_{5}=1 \mathrm{M} \Omega, R_{7}=20 \mathrm{~K} \Omega$ and $C_{2}=C_{3}=$ $1 \mu \mathrm{F}$.

$$
V_{C 3}=-e^{-t}-e^{-2 t}+e^{-t}-e^{-2 t}+1,
$$

from which we can obtain the value of $V_{2}$ if add the gain $\left(R_{7} / R_{6}+1\right)$.

\subsubsection{Noise response}

As for the noise response of the system, the SNR, THD, and SINAD are estimated based on the floor noise. The signal has a fundamental frequency at $1 \mathrm{~Hz}$ and an amplitude of $1 \mathrm{~V}$. It also contains harmonics at $2 \mathrm{~Hz}, 3 \mathrm{~Hz}, 4 \mathrm{~Hz}, 5 \mathrm{~Hz}$, and $6 \mathrm{~Hz}$ with amplitudes of 50, 33, 25, 20 and 16.6 percent respectively, all these magnitudes with respect to the fundamental and additive Gaussian noise with $\mu=0$ and $\sigma=1$ are used to calculate the response to noise. Where SNR is noise signal, THD is total harmonic distortion, and SINAD is signal to noise ratio versus distortion.

As shown in Table III in column SNR, the maximum value is $3 \mathrm{db}$ with a noise amplitude of $s=0.353 \mathrm{Vrms}$.

\section{Conclusions}

In this article, an electronic emulator circuit to a first-order differential equation with time-delay has been developed in a theoretical and experimental manner. Different software tools can be used to find the solution of a first-order differential equation with time-delay. In this article, we used Matlab R14 running on the Ubuntu-Linux platform.

To design and manufactured the suggested electronic circuit, a block diagram in Simulink-Matlab is simulated. The $R_{i}$ and $C_{i}$ values from different blocks: gain, Integrator, and time-delay; are tuned according to Simulink-Matlab and theoretical results. The electronic circuit is made experimentally, and the results are shown in Fig. 6, giving a correspondence with the results by simulation Fig. 5 and the theoretical calculations, Table I.

$\mathrm{AC}$ analysis has been performed through bode diagrams finding cut-off frequencies for the delay block of 7,8 , and 9. The study of the transient response, as well as the stable response, was also carried out using the step function, where the critically damped and overdamped response was found for the delay block. As for the noise analysis, the limit values are given for the noise amplitude at $s=0.353 \mathrm{Vrms}$.

Finally, the electronic prototype is simple to assemble on a protoboard using basic elements. This will allow the student to easily make changes to the key system variables and gain a deeper understanding of physical phenomena modeled with time delay.
1. A. González Sorribes, (2011). Sistemas Dinámicos con Retardos Temporales, Departamento de Ingeniería de Sistemas y Automática, Universidad Politécnica de Valencia.

2. K. Shujaee, B. Lehman, Vibrational feedback control of time delay systems, IEEE Trans. Autom. Control, 42 (1997) 1529. DOI: $10.1109 / 9.649695$

3. E. Fridman, U. Shaked, An improved stabilization method for linear systems with time-delay, IEEE Trans. Autom. Control, 47 (2002) 1931. DOI: 10.1109/TAC.2002.804462

4. E.C. Heyde, Theoretical methodology for describing active and passive recirculating delay line systems, Electron. Lett., 31 (1995) 2038. DOI: $10.1049 / \mathrm{el}: 19951356$
5. G. Groenewold, Noise and group delay in active filters, IEEE Trans. Circuits Syst. I, Regul. Pap., 54 (2007) 1471, DOI: 10.1109/TCSI.2007.900181

6. C. Wijenayake et al., RF analog beamforming fan filters using CMOS all-pass time delay approximations, IEEE Trans. Circuits Syst. I, Regul. Pap., 59 (2012) 1061, DOI10.1109/ TCSI.2012.2185294

7. E. Fridman, Introduction to Time-Delay Systems-Analysis and Control. (Springer2014).

8. G. Munguía and M. GarcAntonioia, Ecuaciones diferenciales con retardo, Memorias de la XVII semana regional de investigación y docencia en matemáticas, 20 (2007) 137. 